\title{
Cinémas
}

Revue d'études cinématographiques

\section{L'Ève future et la série culturelle des « machines parlantes ). Le statut singulier de la voix humaine au sein d'un dispositif audiovisuel}

\author{
Alain Boillat
}

\section{Cinélekta 6}

Volume 17, numéro 1, automne 2006

URI : id.erudit.org/iderudit/016321 ar

https://doi.org/10.7202/016321 ar

Aller au sommaire du numéro

Éditeur(s)

Cinémas

ISSN 1181-6945 (imprimé)

1705-6500 (numérique)

Découvrir la revue

Citer cet article
Résumé de l'article

L'Ève future, oeuvre profondément marquée par l'imaginaire lié à la technique d'inscription des sons et à leur couplage avec l'image, constitue un bon objet pour une réflexion, inscrite dans une perspective d'archéologie du " cinéma » parlant, sur la place et la fonction accordées à la voix dans certains dispositifs audiovisuels. En examinant le roman de Villiers de L'Isle-Adam sous l'angle de la mise en scène fictionnelle de diverses manifestations vocales, on peut définir les spécificités de la voix qui, dans le cas des automates comme au sein du dispositif cinématographique, affirme la présence de l'humain dans la machine. La question du simulacre anthropomorphe permet en effet de s'interroger sur d'inévitables tensions qui résultent de la conjonction de l'humain - le ( grain » de la voix est associé à un individu - et de la machine utilisée pour la (re)production des sons et de l'apparence physique du sujet parlant. À cet égard, la représentation audiovisuelle de l'humain est foncièrement soumise à un phénomène de déliaison dans son rapport à une image animée dont les mouvements ne constituent pas la source effective des sons perçus par l'audiospectateur. Généralement occultée dans le cinéma parlant en raison du primat accordé à l'unicité du sujet-locuteur, cette déliaison fondamentale est associée dans L'Ėve future aux inventions d'Edison, notamment à celles qui permettent de créer l'Andréide. Chez Villiers, l'angoisse provoquée par l'exhibition déshumanisante de la dimension machinique semble à la fois sous-tendre sa description de la

technologie et motiver un dépassement par le recours aux sciences occultes. Or cet argument spirite est symptomatique de l'un des principaux paradigmes de la réception d'une voix enregistrée. Cette dernière induit en effet un régime de ( présence-absence » qui, comme l'a montré Christian Metz, caractérise plus généralement « l’impression de réalité ») au cinéma.

Boillat, A. (2006). L'Eve future et la série culturelle des « machines

parlantes ). Le statut singulier de la voix humaine au sein d'un

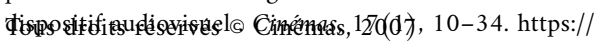

doi.org/10.7202/016321 ar

Ce document est protégé par la loi sur le droit d'auteur. L'utilisation des services d'Érudit (y compris la reproduction) est assujettie à sa politique d'utilisation que vous pouvez consulter en ligne. [https://apropos.erudit.org/fr/usagers/politique-dutilisation/] 


\title{
L'Ève future and the Cultural Series "Talking Machines": The Peculiar Status of the Human Voice in Audio-Visual Technology
}

\author{
Alain Boillat
}

\begin{abstract}
L'Eve future, a profound expression of the way sound recording technologies and their coupling with the image have been imagined, is a good place to begin thinking, from the vantage point of an "archaeology" of sound cinema, about the role and function of the voice in audio-visual technology. In looking at Villiers de L'Isle-Adam's novel, published in English as Tomorrow's Eve, from the perspective of the fictional staging of various vocal manifestations, we can define specificities of the voice which, in the case of both automatons and the film apparatus, create a human presence within the machine. The question of the anthropomorphic simulacrum makes it possible to enquire into the tensions which inevitably arise when the human - the "grain" of an individual's voice_-and the machine are joined to (re)produce the sounds and physical appearance of the speaking subject. In this respect, the audio-visual representation of the human is fundamentally subjected to a kind of decoupling with respect to a moving image, which is not the real source of the sounds heard by the "listener-viewer." In L'Ève future, this fundamental decoupling, which is generally obscured in sound cinema because of the primacy of the subject-speaker's unity, is associated with Edison's inventions, in particular those which made it possible to create his Android. In Villiers's novel, the anxiety brought on by the dehumanizing exhibition of the mechanical dimension appears both to underlie its description of technology and to motivate the use of black magic. This spiritualist argument is symptomatic of one of the principal paradigms of the way a recorded voice is received. The voice creates a sort of "presence-absence" which, as Christian Metz demonstrated, is characteristic in general terms of the cinema's "impression of reality."
\end{abstract}




\title{
L'Ève future et la série culturelle des "machines parlantes ». Le statut singulier de la voix humaine au sein d'un dispositif audiovisuel
}

\author{
Alain Boillat
}

\section{RÉSUMÉ}

L'Ève future, œuvre profondément marquée par l'imaginaire lié à la technique d'inscription des sons et à leur couplage avec l'image, constitue un bon objet pour une réflexion, inscrite dans une perspective d'archéologie du "cinéma" parlant, sur la place et la fonction accordées à la voix dans certains dispositifs audiovisuels. En examinant le roman de Villiers de L'Isle-Adam sous l'angle de la mise en scène fictionnelle de diverses manifestations vocales, on peut définir les spécificités de la voix qui, dans le cas des automates comme au sein du dispositif cinématographique, affirme la présence de l'humain dans la machine. La question du simulacre anthropomorphe permet en effet de s'interroger sur d'inévitables tensions qui résultent de la conjonction de l'humain - le "grain" de la voix est associé à un individu — et de la machine utilisée pour la (re)production des sons et de l'apparence physique du sujet parlant. À cet égard, la représentation audiovisuelle de l'humain est foncièrement soumise à un phénomène de déliaison dans son rapport à une image animée dont les mouvements ne constituent pas la source effective des sons perçus par l'audiospectateur. Généralement occultée dans le cinéma parlant en raison du primat accordé à l'unicité du sujet-locuteur, cette déliaison fondamentale est associée dans L'Ẻve future aux inventions d'Edison, notamment à celles qui permettent de créer l'Andréide. Chez Villiers, l'angoisse provoquée par l'exhibition déshumanisante de la dimension machinique semble à la fois sous-tendre sa description de la technologie et motiver un dépassement par le recours aux sciences occultes. Or cet argument spirite est symptomatique de l'un des principaux paradigmes de la réception d'une voix enregistrée. Cette dernière induit en effet un régime de "présence-absence» qui, comme l'a montré Christian Metz, caractérise plus généralement «l'impression de réalité» au cinéma. 
La possibilité de suivre partout un acteur, de l'œil et de l'oreille à la fois, a semblé rendre nécessaire leur couplage. Sans doute, cette source de servitude est liée à la satisfaction absolue d'une soif de réalisme, de reproduction absolue du monde - comme si nous pouvions encore être présents à son commencement.

Cavell 1999

Une machine pour voir qui s'appelle les yeux, pour entendre les oreilles, pour parler la bouche; j'ai l'impression que c'est des machines séparées, il n'y a pas d'unité. On devrait avoir l'impression d'être unique, j'ai l'impression d'être plusieurs.

Jean-Luc Godard, Pierrot le fou

Le cinéma ne fut pas, loin s'en faut, la première «machine parlante" utilisée à des fins d'exhibition spectaculaire. Inscrit dans une longue filiation d'inventions techniques et de discours portant sur la représentation audiovisuelle de l'humain, le parlant - terme en soi révélateur de la prépondérance accordée à la parole, le véritable «sujet» des films parlants résidant, ainsi que le notait Jean-Louis Comolli (1972), dans le Sujet parlant luimême - a imposé un mimétisme anthropomorphe qui, dans ses principes, est comparable à celui qui sous-tendait la fabrication d'automates à visage humain et certains développements de la technique phonographique. Afin de rendre compte, dans une perspective abordée récemment par des chercheurs tels que Rick Altman, Guisy Pisano ou Édouard Arnoldy, de l'hétérogénéité des modes de sonorisation des films caractérisant la période des "sons du muet", nous proposons d'examiner ces pratiques en élargissant à la sphère de l'audiovisuel la notion de "dispositif" telle qu'elle a été théorisée par François Albera et Maria Tortajada (2004) dans un article programmatique portant sur les «dispositifs de vision ». Proposant une démarche épistémologique qui 
convient à notre objet (puisqu'il importe de s'interroger sur les conditions qui ont favorisé l'émergence d'une conception de l'audiovisuel dominée par le paramètre de la synchronisation vocolabiale), les auteurs conçoivent le dispositif comme une configuration définie par la mise en relation de trois termes: la représentation, la machinerie et le spectateur. Dans notre perspective - qui nécessite de parler d'une représentation et d'une machinerie audiovisuelles, ainsi que d'un audiospectateur-, chacun de ces pôles se voit lui-même scindé en deux faces (l'une visuelle, l'autre sonore). C'est pourquoi il s'agit non seulement de mesurer les implications des interactions résultant de la mise en présence de ces trois termes (relation horizontale), mais aussi d'établir quels sont les critères qui permettent de déterminer le rapport instauré entre les deux facettes de chacun de ces pôles (relation verticale). Il est dès lors possible d'envisager la diversité des pratiques auxquelles certaines technologies peuvent donner lieu en examinant les dispositifs audiovisuels en fonction d'axes de pertinence spécifiques. En ce qui concerne la relation horizontale entre l'audiospectateur et la machinerie, on opérera par exemple une distinction entre plusieurs types de voix (re)produites selon que l'émission est activée par l'usager (kinétophone, poupée-phonographe), par un présentateur (spectacles phonographiques) ou par une instance invisible assimilée à la machinerie. En outre, les relations verticales dues à la corrélation des pans visuel et auditif different d'un dispositif à l'autre. Elles sont notamment fonction des modalités qui caractérisent la synchronisation effectuée à l'aide de la machinerie — ou plus généralement au sein de ce que je propose de nommer «l'espace de production de la représentation" - , opération qui est susceptible de présenter un degré de mécanicité variable. En effet, entre le cas d'une profération live (imputable par exemple à des acteurs dissimulés derrière un écran) et celui d'un couplage d'appareils distincts entièrement assuré par la machinerie, il faut pouvoir considérer également des systèmes semi-mécaniques, à l'instar du "Ciné-pupitre» de Charles Delacommune (1923), qui nécessitait à la fois que des récitants soient présents dans la salle et une synchronisation du défilement de la pellicule avec celui d'une bande comprenant des repères pour la lecture. 
Au regard des combinatoires possibles (dont les « installations» d'artistes contemporains donnent un aperçu), on comprend que le cinéma parlant institutionnalisé gagne à être considéré dans un spectre plus vaste de technologies qui appartiennent, comme lui, à la série culturelle ${ }^{1}$ des machines parlantes. D'un point de vue méthodologique, cette conception permet d'affranchir l'étude historique des impératifs de périodisation, puisqu'une série peut certes dériver d'une autre, mais aussi lui faire lointainement écho ou se développer parallèlement à des séries voisines. La contamination réciproque peut d'ailleurs s'exercer en raison d'une contiguïté spatiale qui, résultant de pratiques spectaculaires non fixées, est elle-même soumise à d'importantes variations diachroniques et à des contingences diverses. Rick Altman (2004, p. 80) fait mention de plusieurs phénomènes de ce type, par exemple lorsqu'il précise qu'en 1894-1895 les lieux d'exploitation du kinétoscope comprenaient, outre l'appareil de visionnement d'Edison, des salons où des phonographes étaient mis à la disposition du public sous forme de machines à sous. Par conséquent, ces divertissements fournissaient séparément ce que l'on retrouvera conjointement dans les représentations offertes ultérieurement par le kinétophone. Pour prendre un exemple spécifiquement lié à la voix humaine, on peut supposer que l'enthousiasme du peintre Léopold Robert face à l'interactivité proposée par la voix acousmatique de "La fille invisible ${ }^{2}$ ", spectacle présenté en 1815 par le célèbre fantasmagore Robertson, s'explique par le fait que cette curiosité côtoyait, dans le même cabinet, une exposition d'automates parlants ${ }^{3}$. Convergences et divergences entre séries permettent de mieux saisir la singularité de chaque dispositif.

L'historien du cinéma Jacques Deslandes (1966, p. 63) a souligné le fait que le cinématographe a coexisté avec certaines «machines parlantes»: "Le cinématographe animait des androïdes aphones. Le rayon des jouets allait plus loin que lui car on trouvait alors dans le commerce des poupées qui parlaient et chantaient. " Deslandes procède simultanément à une différenciation - le passage de la salle de spectacle au grand magasin implique un changement de paradigme - et à une corrélation qui repose sur le terme "androïde" appliqué aux êtres de l'écran. 
En valorisant les jouets, l'historien met l'accent sur une dimension spécifique du mimétisme: si ces petites machines «allaient plus loin» que le cinématographe, c'est dans le sens de l'anthropocentrisme du "double magique " qu'Edgar Morin (1958) décrivait comme l'un des besoins majeurs de l'homme sur le plan de l'imaginaire. Les tentatives des inventeurs et pionniers pour adjoindre une voix aux images animées s'expliquent souvent par un fantasme similaire.

Lorsque le phonographe, breveté en 1877 par Edison, connut ses premières présentations et que les commentaires à son sujet se multiplièrent dans l'espace public, il était couramment dénommé "machine parlante", une expression qui met en évidence la composante orale de sa "performance" (technologique, mais aussi spectaculaire lorsqu'il était exhibé publiquement), et non sa qualité d'appareil inscripteur permettant d'effectuer une "écriture" des sons. Cette désignation faisait donc office d'hyperonyme, inscrivant dans une même série culturelle des dispositifs fort différents. Le recours à un terme préexistant indique que les contemporains de l'invention d'Edison établissaient une filiation évidente entre le phonographe et certaines machines à représentation antérieures. Dans le cadre d'une réflexion de type épistémologique portant sur les grands paradigmes de la réception du "cinéma parlant" qui prévalurent trois ou quatre décennies avant la standardisation de ce dernier ${ }^{4}$, il nous paraît pertinent de comparer certains domaines d'activité qui, à la fin du XIXe siècle, étaient particulièrement perméables. La frontière était en effet souvent ténue entre le spectacle conçu à des fins de divertissement et la démonstration scientifique (notamment dans le domaine de la physiologie), ou entre les sciences exactes et le spiritisme. Nous nous contenterons d'aborder une catégorie discursive particulière: la fiction littéraire basée sur des spéculations technologiques. Ainsi que l'a montré Charles Grivel (1992), l'imaginaire de la reproduction mécanique d'un locuteur, dont on trouve notamment une trace en 1748 sous la plume du philosophe Julien Offroy de La Mettrie, se voit ravivé dans le champ de la littérature avec la diffusion des inventions d'Edison et de Berliner durant le dernier quart du XIX siècle. 
L'une des fictions analysées par Grivel qui témoigne le mieux des mutations qui s'opèrent autour des années 1880 est le roman L'Eve future, publié en 1886 par Villiers de L'Isle-Adam ${ }^{5}$, auteur qui fut par ailleurs un proche ami de Charles Cros, inventeur et poète français qui déposa à l'Académie des Sciences, huit mois avant l'obtention du brevet par Edison, une lettre scellée dans laquelle il détaillait de façon plausible le fonctionnement du procédé phonographique. Bien que souvent commentée dans ses rapports avec le cinéma, L'Éve future est une œuvre extrêmement riche dont le potentiel herméneutique est loin d'avoir été épuisé. Ce récit, dans lequel un Edison mythifié en "papa du phonographe» (titre que Villiers donne au chapitre 2 du livre premier) met son invention au service de la reproduction mécanique d'un être parlant, s'avère propice à une discussion du statut de la voix au sein d'un dispositif audiovisuel - et notamment de la voix enregistrée, puisque le romancier met l'accent sur la nature indiciaire de l'enregistrement phonographique. Cette question fréquemment soulevée (notamment chez Burch, 1990, p. 33-55) a été somme toute peu approfondie par les exégètes de l'œuvre de Villiers qui se sont attachés à la dimension technologique du monde représenté, soit parce qu'ils tendent à privilégier l'image, soit parce que les diverses manifestations vocales représentées dans le roman ne sont pas considérées dans leur singularité. Il est symptomatique qu'André Bazin (1985, p. 23), lorsqu'il évoque succinctement le roman de Villiers pour illustrer "le mythe du cinéma total», ne se réfere nullement à l'androïde proprement dit (bien que Hadaly résulte de la conjonction d'une apparence humaine et d'une parole), mais se borne à évoquer la projection de vues animées effectuée par l'Edison fictif à l'aide d'un lampascope ${ }^{6}$. Quoique l'extrait qu'il cite se termine sur le chant de la danseuse, Bazin évacue la question du couplage de l'image et de la voix. Dans un article consacré aux simulacres anthropomorphes créés grâce à la technologie audiovisuelle, Tom Gunning (2001, p. 22) revient (de façon fort peu critique) sur Bazin et cite au passage l'œuvre de Villiers, qu'il n'aborde pas en raison des nombreuses analyses auxquelles, selon lui, elle a déjà donné lieu. En dépit de la référence récurrente à $L ' E v v e$ future dans les études qui examinent la 
représentation des sciences dans les récits littéraires d'anticipation, ce roman mérite selon moi d'être étudié plus avant sous l'angle de la composante vocale et des implications de la technologie phonographique. Comme nombre de commentateurs l'ont souligné, il s'agit en effet d'une œuvre où $l a V_{o i x}{ }^{7}$ constitue une préoccupation fondamentale.

\section{Les voix du roman}

L'importance de la voix apparaît dans L'Ève future dès le monologue inaugural d'Edison. L'inventeur se lamente de n'avoir pu enregistrer toutes les voix du passé, notamment celle de Dieu qui, pourrait-on dire, lui aurait permis de faire de l'épreuve (phonographique) une preuve (ontologique). Par conséquent, la voix est placée d'entrée de jeu sous le signe de l'absence ou de la disparition des êtres - situation qui concerne également Dieu lui-même, en quelque sorte supplanté par l'homme de science qui lui adresse un défi sacrilège. La fonction qui consiste à remédier à une "absence" n'est certes pas propre aux usages de la voix dans l'histoire des dispositifs audiovisuels (ni la question de la "présence-absence" induite par toute représentation, sur laquelle nous reviendrons ci-dessous), puisque l'image photographique fut, elle aussi, destinée en priorité à un rôle d'archivage, relayant par ailleurs sur un mode nouveau le portrait pictural dans sa capacité à conserver une trace des êtres disparus. Toutefois, l'exemple de la voix divine est révélateur de la réception quasi mystique des phénomènes vocaux dépourvus d'incarnation physique, associés tant aux origines divines du monde ("au commencement était le Verbe») - cette association explique probablement la fréquente utilisation au cinéma de la voix over dans une fonction d'instauration inaugurale de l'univers filmique — qu'aux manifestations d'une instance qui se soustrait à l'ordre du représentable.

Dans L'Evve future, le soliloque du savant préfigure le traitement réservé à la voix dans l'ensemble du roman. D'une part, en tant qu'énonciation diégétisée, le monologue est travaillé sur le plan des registres de langage et des procédés d'inscription de l'oralité dans les discours en style direct; d'autre part, la voix est 
exploitée en tant que motif narratif. Il est pertinent de souligner, à l'instar de Gwenhaël Ponnau (2000, p. 127), que «thématiquement, mais aussi poétiquement et structurellement, L'Ève future peut apparaître comme le roman de la voix ou plutôt des voix». Toutefois, Ponnau examine la structure polyphonique et les diverses "didascalies" du roman sans tisser de liens entre de telles caractéristiques stylistiques et la composante technologique inscrite au cœur du récit. Il est pourtant éclairant d'établir une corrélation entre la représentation de la voix et la problématique des machines parlantes figurées par Villiers. En effet, non seulement son roman décrit fréquemment la voix des personnages féminins, et ce avec force nuances (timbre, intensité, intonation, etc.), mais il convoque également diverses voix entendues en situation d'écoute acousmatique, c'est-à-dire dans des cas où la source de la voix ne peut être vue par les personnages. De telles manifestations vocales sont motivées dans l'univers diégétique soit par des procédés de communication à distance (télépathie ou téléphonie), soit par la restitution d'un son émis préalablement (phonographie). Les sons du premier type interviennent dès le début de L'Ève future: l'employé et le fils d'Edison sont exclusivement présentés au lecteur sous la forme de voix. Dans l'écheveau d'interventions verbales transcrites en discours direct, l'expression "une voix" désigne d'ailleurs souvent par synecdoque les différents personnages ${ }^{8}$. La voix est donc associée à une absence que le phonographe - à l'instar de l'Andréide elle-même, qui se substitue, en tant qu'idéalisation, à la femme réelle — s'emploie à combler partiellement. Isolé dans son laboratoire, Edison dédaigne de s'adresser directement à ses interlocuteurs, se contentant, en guise de réponse, d'actionner un phonographe relié à un téléphone (Villiers 1993, p. 53). Ainsi son rapport aux autres est-il fortement médiatisé par les techniques de reproduction et de transmission de la voix. Ce comportement quelque peu asocial est sans doute un vestige de l'intention initiale de Villiers qui consistait à dresser un portrait sarcastique de l'homme de science. Cependant, le roman multiplie les occurrences d'une voix over dont la source est située dans un autre lieu (celle de la dormeuse Any Anderson), voire dans un espace-temps différent 
de celui de l'auditeur (lorsque Hadaly répète les mots d'Alicia), soulignant ainsi la dissociation et la désincarnation produites par les dispositifs d'Edison. La séparation de l'être physique et de la parole est d'ailleurs explicitement thématisée dans le discours de l'inventeur, qui prétend pouvoir conserver le corps d'Alicia tout en modifiant son âme, donc satisfaire au souhait de son ami («qui m'ôtera cette âme de ce corps!», s'exclame lord Ewald à cinq reprises). Cette dichotomie fondamentale permet de distinguer les dimensions verbale et vocale de l'acte illocutoire, le jeune lord disant de son amante que "ses paroles paraissaient dépaysées et gênées dans sa voix» (p. 78). En outre, une même logique oppositionnelle (corps/âme et voix/parole) régit les jugements de valeur émis à propos des attributs vocaux. On observe en effet dans le portrait que Villiers fait d'Alicia - il n'est à cet égard pas anodin qu'elle soit cantatrice, une profession qui est le lieu d'exercice d'une performance vocale fortement ancrée dans l'expressivité humaine et dans le régime de l'oralité, tout comme le sera la Stilla dans Le château des Carpathes de Jules Verne $(1978)^{9}$ - un contraste entre la perfection de l'inné et la médiocrité de l'acquis: tout ce qui ne relève pas de son «essence» est entaché par de basses aspirations de philistin. Il précise par exemple que la jeune femme s'exprime "avec une intonation de patronne de magasin, mais, aussi, avec un timbre de voix d'une limpidité idéale» (p. 271). La captation de la voix est donc présentée comme une entreprise d'extraction d'une partie du réel qui, assemblée avec un élément nouveau, contribue à créer l'idéal féminin.

\section{La représentation machinique en tant que dispositif audiovisuel : l'Andréide et son spectateur}

En faisant du phonographe l'invention-clé dont il est possible de dériver diverses applications, Villiers inscrit sa machine parlante dans le contexte de l'intermédialité (au sens d'Altman, 1989) ${ }^{10}$. En effet, l'élaboration de l'Andréide, décrite avec une précision fétichiste, fait également appel à plusieurs modalités de l'image projetée et, de la sorte, se voit présentée comme le produit d'une représentation visuelle qui excède la seule présence physique de l'automate. Lorsque Villiers (1993, 
p. 120) précise que son Edison entend surpasser les fabricants d'automates tels que Vaucanson ou Maelzel, il inscrit son personnage dans la filiation de ces constructeurs tout en lui accordant une supériorité que lui confère la maîtrise de la technique phonographique. Car si la machinerie visuelle n'intervient pas à proprement parler dans le fonctionnement de l'être mécanique, elle est nécessaire à la conception de son enveloppe corporelle et, surtout, à l'établissement d'un synchronisme entre les paroles et les gestes. Edison utilise en premier lieu un dispositif de projection dont les "photographies successives" lui permettent de montrer à lord Ewald l'image animée d'une belle danseuse. Afin de prouver à son ami combien l'art de la toilette peut leurrer les hommes (p. 199), il compare ensuite cette image avec la dépouille de la même femme. La projection n'est pas muette: la danseuse chante, et Villiers précise que le "mouvement labial» est également reproduit, ce qui laisse supposer un synchronisme audiovisuel. Il est intéressant de constater qu'à aucun moment le statut illusoire de la représentation audiovisuelle ne se trouve mis en cause par les personnages qui assistent au spectacle, quand bien même la démonstration vise précisément à révéler l'apparence trompeuse du sujet filmé. L'illusion de la représentation produite par le dispositif est en quelque sorte déplacée sur la nature du représenté, selon un processus qui s'apparente à un phénomène d'immersion du spectateur de cinéma dans la diégèse filmique. Les spéculations scientifiques du romancier révèlent que ce dernier postule une fidélité totale de la reproduction audiovisuelle. Bien qu'il exhibe dans des parties descriptives le fonctionnement des appareils manipulés par Edison, Villiers tend en effet à occulter la dimension représentationnelle des voix phonographiées en soulignant de façon récurrente leur perfection. Ainsi la locutrice ne tient-elle aucunement compte de la situation d'enregistrement lorsqu'elle s'exprime — la voix d'Alicia est fixée à l'insu de cette dernière —, comme si l'optimisation des qualités de la reproduction était indépendante des conditions "prophonographiques ${ }^{11}$ "; de plus, le fonctionnement de l'appareil ne semble exercer aucune influence sur le rendu de la voix. Cette conception, également décelable chez Jules Verne ${ }^{12}$, 
selon laquelle la technologie de reproduction du son n'est absolument pas perceptible en tant que telle (surtout lorsqu'on l'applique à la voix, c'est-à-dire lorsqu'il s'agit de respecter l'individualité d'un locuteur en bannissant toute intrusion de la mécanicité), informera tout un pan de la réflexion menée sur l'audiovisuel au cours du $\mathrm{XX}^{\mathrm{e}}$ siècle. Dans cette perspective, la représentation passe pour une pure restitution, ce qui suppose que l'opération de transmission n'implique aucune transformation. Cette conception atteindra son paroxysme lorsque l'industrie mettra sur le marché des systèmes labellisés high fidelity ${ }^{13}$.

L'usage qu'Edison fait du lampascope - appareil que Villiers mentionne également dans un conte satirique intitulé "L'affichage céleste ${ }^{14}$ " - assigne à lord Ewald une position d'audiospectateur. On peut dire que les expériences proposées par l'inventeur sont présentées sous la forme de dispositifs. Par la suite, les interactions de l'Andréide avec lord Ewald sont significativement qualifiées à plusieurs reprises de "scènes». Avant de succomber à l'illusion, l'aristocrate s'indigne en ces termes de l'ambition démesurée d'Edison:

Quel homme s'est imaginé que ce sinistre automate pourrait m'émouvoir à l'aide d'on ne sait quels paradoxes inscrits sur des feuilles de métal! [...] Et quel risible orgueil semble concevoir d'électriques fantômes qui, vêtus de la forme de la femme, prétendent se mêler à notre existence? - Ah! ah! ah! Mais j'oubliais! je suis au théâtre! Et je ne dois qu'applaudir. La scène est, en effet, bien étrange! (Villiers 1993, p. 319).

Grâce aux feuilles d'étain qui défilent sur le cylindre des phonographes de la machine (ses "poumons d'or", expression qui les hisse au rang de précieux organes vitaux), la discussion constitue une sorte de performance scénique. La relation qui s'établit entre Edison et son invité relève d'un contexte spectaculaire qui comprend l'objet de l'exhibition (l'automate) et les deux pôles de la communication (le promoteur et le spectateur). Dans ce passage, lord Ewald qualifie le contenu des inscriptions phonographiques de "paradoxes": la production artificielle d'une interaction verbale est jugée invraisemblable, même au sein de la fiction. Cet élément, nodal au niveau narratif 
- Edison différant sans cesse son explication - , constitue une étape décisive dans la genèse du roman, puisque le dénouement ne s'est imposé à Villiers qu'après plusieurs années. L'éclaircissement ultime du fonctionnement de l'Andréide correspond en fait à l'achèvement de l'œuvre littéraire, qui se présente comme une réécriture du livre de la Genèse: écriture et phonographe sont les instruments d'une répétition de l'acte fondateur, le don de la parole se présentant comme le garant de l'humanisation de l'artefact. C'est pourquoi Edison et le roman lui-même font de ce don leur dessein ultime: "La voix est le centre de gravité du roman, comme le poème d'Hadaly est sa finalité ", écrit Catherine Coquio (1999, p. 47). Si le silence a le dernier mot (puisqu'il s'agit littéralement du dernier mot du livre), c'est parce que l'origine divine s'avère in fine indépassable.

Quant à l'image, elle intervient dans L'Éve future à travers le façonnage de Hadaly qui s'élabore sur la base d'une série de repères identifiés grâce à des "épreuves photographiques " (Villiers 1993, p. 248). L'apparence physique de l'être artificiel est le résultat d'une série d'images analogues à celles qu'obtiendra Étienne-Jules Marey à l'aide de sa technique chronophotographique $^{15}$. Cet élément de la fiction suggère une possible connexité entre la série des automates et celle des appareils de projection de vues animées. L'image projetée intervient dans l'élaboration d'une machine qui, une fois achevée, ne garde pas trace du système de projection qui a servi à sa conception. L'Edison de Villiers motive de telles expériences préalables en soulignant la nécessité d'une corrélation entre le tracé des mouvements corporels inscrit sur un cylindre - outil lui-même parent du procédé phonographique - et le sillon résultant de l'enregistrement de la voix. Il dit de ce processus qu'il consiste à établir des "correspondances expressives", et le commente ainsi : «Ne faut-il pas, en effet, que le mouvement des deux phonographes, uni à celui du cylindre, produise l'homogénéité du geste et de la parole ainsi que du mouvement labial ?» (p. 217). Cette explication témoigne de l'importance du synchronisme vocolabial sur lequel repose, au cinéma, ce que Dominique Chateau (1986, p. 131) nomme de façon dépréciative le « dogme du réalisme audiovisuel ». 
En dépit de la perfection de la création menée à bien par l'Edison de son roman, Villiers met en évidence l'irréductibilité de la parole à la machine, cette dernière étant foncièrement privée d'âme. Le mouvement qui caractérise la projection chronophotographique anime certes les choses, mais seule la voix " anime" véritablement les êtres. Or, selon Villiers, cette origine de la voix ne peut être recréée par la seule maîtrise des lois de la mécanique et de l'acoustique: chez lui, Edison est également magnétiseur.

\section{L'invention technique parachevée grâce à une intervention spirite}

La double désincarnation résultant du couplage du phonographe et du téléphone opéré par Edison lorsqu'il communique avec l'extérieur de Menlo Park se voit réitérée lorsque intervient Sowana, puisque l'inventeur entre en contact avec elle en utilisant simultanément ses dons de télépathie et un combiné téléphonique (Villiers 1993, p. 333). La mise en relation de ces deux pratiques est l'indice des similitudes importantes que présentent, dans l'imaginaire de l'époque, la technologie et certains champs du savoir que l'on qualifierait aujourd'hui de "parascientifiques ".

Cette présence de l'occultisme nous rappelle que la thématique technologique ne doit pas éclipser la face symboliste de l'œuvre de Villiers, dans laquelle l'«électricien " Edison possède également des pouvoirs télépathiques qui font basculer le récit dans le surnaturel. Le recours à des procédés spirites pour doter la machine de la parole montre combien Villiers considère l'expression verbale comme totalement incompatible avec la composante mécanique. La singularité de la voix concerne autant son appartenance indéfectible à un individu (en dépit du péril que la phonographie peut constituer à cet égard) que l'étrangeté suscitée par son apparition.

Chez Villiers, la distinction entre l'humain et l'inhumain repose partiellement, en ce qui concerne la voix, sur une opposition entre production et reproduction. L'origine à la fois occulte et technologique de la voix reproduite est révélatrice, comme l'oxymoron du titre "L'Ève future», de ce paradoxe du 
symbolisme qui réside dans la collusion du mythologique (le mythe adamique de l'origine) et du scientisme. Bien que Villiers entendît initialement pourfendre le positivisme comme il l'avait fait dans certains de ses contes, la version finale de son roman témoigne d'une évidente attirance pour le scientisme, en dépit de la toute fin du récit qui souligne la vanité de l'entreprise prométhéenne. Or la dimension technologique de cette entreprise peut être envisagée dans le cadre des phénomènes de déliaison entre la voix proférée et le corps visualisé tels qu'ils peuvent avoir lieu dans des films parlants, où l'expression vocale semble momentanément se desquamer de son origine humaine, sinon en révélant sa nature d'enregistrement, du moins en dévoilant l'artifice du synchronisme vocolabial.

En effet, le personnage de miss Alicia se caractérise par une «disparité totale» entre sa personne physique et sa personne morale (Michelson 1984, p. 7), tandis que sa reproduction "audiovisuelle», résultat d'opérations complexes assurant la synchronisation des divers mécanismes, donne lieu à une fusion de la beauté physique du modèle et de la perfection intérieure dont il est dépourvu. L'utopisme technologique de l'œuvre réside donc plus profondément dans une conception unitaire de l'Andréide qui fait de celle-ci une illusion complète de la vie.

Vers 1880, une publicité pour un spectacle d'automates prenait soin de souligner combien l'invention qui était exhibée se distinguait avantageusement des appareils de reproduction: «Ne pas confondre cette merveilleuse machine, unique au monde, avec le phonographe qui n'est qu'un simple effet d'ÉCHO ${ }^{16}$. » Ainsi, le fait que la parole soit produite sans enregistrement préalable constitue-t-il un argument promotionnel, bien que ce procédé ne soit aucunement une nouveauté. De même, Villiers met en évidence les limites de la reproduction phonographique, postulant quant à lui le dépassement de cellesci par les «techniques» de l'occultisme: l'esprit de Sowana, qui s'exprime à travers la bouche de l'Andréide, permet l'intrusion de l'aléa qui caractérise la conversation courante, et donc l'interaction avec l'audiospectateur. Un point commun apparaît toutefois entre spiritisme et phonographie, puisque Sowana est réduite à une voix désincarnée, dissociée de mistress Anderson 
qui, dans un état de quasi-catalepsie, lui donne vie. Si l'on met en relation cette intervention du surnaturel avec les diverses inventions d'Edison consistant à séparer la voix du corps, on comprend ce qui, dans le phonographe, pouvait être conçu comme une ambition démiurgique à la fois attirante et périlleuse. Dans L'Ève future, la rencontre d'une voix en déshérence avec un corps artificiel voué à la mécanicité du phonographe est présentée comme la garante de la complétude audiovisuelle. Il est significatif que la pierre angulaire de l'utopie scientifique du romancier relève du "magnétisme psychique": il faut à Villiers le secours du surnaturel pour conjurer l'angoisse de l'absence suscitée par le phonographe, pour échapper à la monstruosité que représente une voix phonographique détachée de toute âme - une voix totalement déshumanisée comme celle que met en scène Marcel Schwob dans La machine à parler (1920) ${ }^{17}$. Cet élément du récit s'inscrit plus largement dans la mouvance symboliste qui dénonce le matérialisme de la société fin de siècle (incarné chez Villiers par l'être aimé qu'il s'agira de remplacer par une machine) tout en proposant de retrouver l'âme perdue, fût-ce, comme dans le cas à cet égard particulièrement ambivalent du roman L'Ève future, en recourant au produit même de l'industrie.

Si la technique provoque sur le spectateur des effets qui peuvent être associés à des phénomènes "magiques» (Villiers qualifie Edison de "magicien du siècle" ou de "sorcier de Menlo Park»), elle peut aussi se mettre au service de pratiques spirites. James Lastra (2000, p. 224, note 3) remarque à cet égard que les transformations provoquées par les technologies de la photographie, de la phonographie et de la télégraphie touchèrent également le discours et les méthodes du spiritisme. En 1673 déjà, Athanasius Kircher (1966, p. 161) rappelait que tout un courant de commentateurs des "têtes parlantes" avait interprété les voix émises par ces créatures comme étant démoniaques, car le diable se serait manifesté sous la forme d'une voix proférée par des statues. Chez Villiers, les créations artificielles douées de parole sont étroitement liées à la croyance au surnaturel: grâce à Edison, le fonctionnement "moderne" de l'Andréide se fait l'héritier de pouvoirs médiumniques 
ancestraux. Cette contamination du technologique par le merveilleux semble liée à la polysémie même du terme "médium": il n'y a qu'un pas de la médialité à la médiumnité, de l'ici matériel du mécanisme à... l'au-delà. On rappellera d'ailleurs que (le vrai) Edison, bien que n'étant nullement un adepte de la "cause", entreprit dans les années 1920 d'améliorer les «tablettes Oui-Ja» utilisées par les médiums, développant à cette fin un appareil électrique enregistreur dont le système d'amplification était particulièrement sensible.

La cristallisation de préoccupations à la fois technologiques et parascientifiques autour des phénomènes vocaux constitue l'un des principaux contextes culturels qui sous-tend, chez Villiers, la représentation de l'automate parlant. Alors qu'Arthur Conan Doyle (1981, p. 315) consacrera, quarante ans après Villiers, un chapitre de son Histoire du spiritisme à des faits "permanents et patents qui exigent maintenant des savants, des artistes et des moqueurs une solution au mystère de leur production ", l'auteur de L'Eve future met en scène une machine destinée à fixer les paroles de l'être aimé et, dans son "avis au lecteur", adresse son roman aux «rêveurs" comme, précisément, aux "railleurs». On le voit, la prise en compte des discours sur l'occultisme permet de souligner la perméabilité de domaines du savoir aujourd'hui nettement différenciés ${ }^{18}$.

\section{La présence-absence du «signifiant imaginaire »}

Il est significatif que les inventions de l'Edison de Villiers ne visent jamais à produire une voix — comme l'eût permis un appareil plus fantaisiste - , mais toujours à reproduire une émission préalable dont l'auteur est absent. Ce constat vaut d'abord pour les oiseaux artificiels qui tiennent compagnie à l'Andréide dans son antre: alors que l'on aurait plutôt associé ces simulacres animaliers à la tradition des automates capables de produire mécaniquement de la parole, ils restituent dans L'Ėve future les commentaires des visiteurs de Menlo Park enregistrés par le propriétaire du lieu (Villiers 1993, p. 168). Il en va ensuite de même de la reproduction de la voix de miss Alicia Clary, puisque Edison, qui projetait de graver des cylindres durant l'une des représentations de la comédienne, l'invite chez 
lui, prétextant une répétition, pour lui faire prononcer ce que dira l'Andréide.

Limportance du ça a été sonore apparât chez Villiers à travers l'examen des rapports que l'auteur établit entre la représentation, la machinerie et l'audiospectateur. On peut postuler que l'automate Hadaly déclenche chez lord Ewald une impression de réalité à certains égards comparable à celle que produit le dispositif du cinéma sur son spectateur. Le pacte faustien qui lie le lord à l'inventeur se comprend dès lors comme un contrat de lecture portant sur les conditions nécessaires à l'«impression de réalité» étudiée par Christian Metz (1993, chap. 3). En effet, le terme de "présence-mixte» par lequel l'Edison de Villiers (1993, par exemple p. 121 et p. 132) qualifie l'Andréide me semble faire écho à la conception de Metz (1993, p. 64) selon laquelle le signifiant cinématographique «se rend présent sur le mode de l'absence». On peut soutenir que, à l'instar du «signifiant imaginaire" du cinéma, l'Andréide consiste en une "projection" au sens psychologique du terme, puisqu'elle ne doit son existence - et le contrat passé entre l'inventeur et le suicidaire épris de la cantatrice le stipule explicitement - qu'à l'acceptation crédule de l'illusion de la part du spectateur. Indépendamment du contexte technologique (qui prime par contre dans Le château des Carpathes, où un leurre initial renforce la croyance de Franz de Télek) ${ }^{19}$, l'animation de l'être artificiel exprime métaphoriquement la puissance de l'imagination déployée par l'homme sensible dans sa quête de l'Idéal. D'ailleurs, on trouve une thématique similaire dans le conte merveilleux de Villiers intitulé "Véra» (1983) qui, lui, ne fait aucune référence aux sciences positives de l'époque. Dans ce texte, une "voix lointaine" appelle l'homme qui a perdu son épouse, mais tout disparait dès l'instant où, tel Orphée, le mari commet l'irréparable en se souvenant du décès de l'être aimé. En affirmant dans cette nouvelle que «les Idées sont des êtres vivants» (p. 66), Villiers recourt au surnaturel pour évoquer une "présence-mixte» analogue à celle de l'Andréide. En outre, le rapport du spectateur à la représentation s'établit également sur le mode de la fusion, l'être idéal se réalisant dans le Même: «Et ils s'aperçurent, alors, qu'ils n'étaient, réellement, qu'un seul être" (c'est moi qui 
souligne) (p. 67). Dans L'Ève future, Edison précise que l'Autre est créée à l'image du Je, que les paroles apparaîtront telles que désirées par le lord: "Ses paroles ne décevront jamais votre espérance! Elles seront toujours aussi sublimes... que votre inspiration saura les susciter"(Villiers 1993, p. 220). Hadaly résout donc ce paradoxe du phonographe qui, selon Charles Grivel (1992), réside dans l'association de la reconnaissance d'une identité propre (la voix objectivée) avec l'expression du "Je est un autre» rimbaldien (la voix restituée étant différente de celle que l'on croit posséder, la mécanisation dissolvant le sujet). En soumettant la voix de la machine à la voix d'un esprit, Villiers conserve le pouvoir fantasmatique de la mimesis «totale» tout en supprimant l'anxiété provoquée par la mécanisation.

Dans L'Ève future, la voix permet indéniablement de créer l'illusion d'une présence: la cantatrice cache sous la perfection de ses capacités vocales les carences de son "âme»; Edison recourt à l'hypnotisme en dialoguant avec Alicia (Villiers 1993, p. 275); lors de la conversation dans le parc, lord Ewald confond son amante avec l'être artificiel parce qu'il est leurré par les paroles de Hadaly. Ce pouvoir illusionniste n'engage pas nécessairement la composante technologique, mais met plutôt en cause, sur le mode de la régression, une activité psychique caractéristique des tout premiers temps de l'être vivant dont la perception, focalisée sur la voix maternelle, est d'abord et avant tout d'ordre acoustique $^{20}$. Le phonographe apparaît alors comme un instrument destiné à objectiver une pulsion que Denis Vasse (1974, p. 77) constate chez le nourrisson, et qui consiste à utiliser sa propre voix comme un moyen de reproduire imaginairement, sur la base de "traces mnésiques" (concrétisées dans L’Ève future par les sillons du cylindre), la présence de la mère absente: "Par cette activité, l'enfant confond la présence effective du corps de l'autre et le désir qui la rend imaginairement présente: il se fait autre pour mettre en échec l'augmentation douloureuse de la tension et le déplaisir qu'elle lui procure.»

Avec le phonographe, le sujet n'a plus besoin de s'aliéner pour satisfaire son fantasme, puisque la machine se fait elle-même le support de l'altérité. Pour qualifier cette activité qui relève de la représentation, Vasse (1974, p. 78) reprend la formule de Freud 
en disant que «le principe de plaisir l'emporte sur le principe de réalité ". La voix constitue donc l'un des principaux accès à l'imaginaire. Le roman de Villiers convoque deux types d'imaginaire : celui qu'Edison offre au personnage du lord à l'intérieur de l'univers fictionnel et celui, propre à l'époque de l'émergence de la technique phonographique, qui nourrit la fiction. Ce sont ces deux aspects qui sont mis en question dans certains écrits théoriques sur le cinéma, d'une part parce que l'imaginaire est inscrit au cœur du "dispositif» dont traite Metz (1993, p. 10) à partir des postulats lacaniens, d'autre part parce que, comme le dit ce dernier dans Le signifiant imaginaire, "tous les films reposent dès leur signifiant sur l'imaginaire premier de la photographie et de la phonographie».

L'Éve future constitue l'un des nombreux discours à partir desquels se propagea un imaginaire phonographique qui n'a pas été sans marquer certains cadres interprétatifs qui informèrent la réception des divers types de sonorisation appliqués au "cinéma». La réflexion sur la synchronisation audiovisuelle et sur l'«animation" par la voix (qui insuffle une âme à la machine) que l'on peut dégager du roman nous conduit à nous interroger sur la fracture qu'occasionne le couplage de la voix enregistrée et de la figuration d'un locuteur, notamment du point de vue de l'effet produit sur l'audiospectateur. Cette déliaison qui menace parfois de s'afficher au détriment de l'idéologie réaliste dominante confronte le spectateur à la machinerie, pôle du dispositif généralement refoulé par une représentation que l'on veut anthropoïde. En considérant chez Villiers l'automate parlant ainsi que l'exposition des différentes phases de son élaboration comme autant de productions spectaculaires, on appréhende combien la diversité des interactions est grande entre les trois pôles du dispositif. L'intervention ultime de l'occultisme suggère par contre une propriété inaliénable de la voix, dont l'émission est foncièrement associée à l'humain. On ne s'étonnera donc pas du recours fréquent à des voix déliées, surgies d'outre-tombe, que l'on observe dans des films qui visent à créer un certain malaise chez le spectateur en soulignant le caractère dissocié de ce qui est perçu. On retrouve là cette angoisse suscitée à la fin du XIX siècle chez les premiers auditeurs du phonographe, confrontés à une

L'Ève future et la série culturelle des "machines parlantes". Le statut singulier de la voix humaine au sein d'un dispositif audiovisuel 


\title{
voix ostensiblement désincarnée dont saura s'emparer la fiction cinématographique pour la mettre en scène, à l'instar de Villiers dans L'Eve future.
}

\author{
Université de Lausanne
}

\section{NOTES}

1. Nous empruntons le concept de "série culturelle» à André Gaudreault (1997), qui désigne ainsi les pratiques spectaculaires à la croisée desquelles s'est constitué le cinéma des premiers temps.

2. «On fait n'importe quelle question très doucement, on met l'oreille à ce tube et on entend une voix qui sort de ce globe et qui répond toujours et très juste, et (ce qu'il y a de plus étonnant) qui vous fait le portrait de votre personne, qui vous dit quel costume, quel âge vous avez» (Léopold Robert, cité dans Clair 1994, p. 441). J'utilise le terme "acousmatique» dans le sens que lui donne Michel Chion depuis une vingtaine d'années (voir par exemple Chion 2004, p. 411).

3. "Entre autres choses curieuses, nous vîmes un mannequin d'enfant en cire, qui prononçait très bien toutes les lettres de l'alphabet. La manière dont il s'exprimait pour prononcer plusieurs mots de suite était très intelligible. C'est une chose vraiment surprenante» (lettre de Léopold Robert à son frère, citée dans Chapuis et Droz 1949, p. 331).

4. Cette standardisation correspond à la généralisation du «son-sur-film», exacerbation du principe de la «fixation» qui caractérise le devenir mécanique que connaît "l'oralité» (au sens large que lui donne le médiéviste Paul Zumthor) à partir de l'essor de la technique phonographique; voir Lastra 2000 ainsi que Pisano et Pozner 2005 (actes d'un colloque dont la plupart des intervenants ont travaillé dernièrement sur ce type de problématique).

5. En fait, ce roman fut ébauché dès 1877 (sous le titre L’Andréide paradoxale), puis il parut en feuilleton dans une version inachevée intitulée L'Ėve nouvelle, avant de connaître une version définitive livrée du 18 juillet 1885 au 27 mars 1886 dans l'hebdomadaire La Vie moderne.

6. Le lampascope est un type de lanterne magique en fer-blanc coloré servant traditionnellement à la projection de plaques de verre peintes grâce à une lampe à pétrole et à un objectif.

7. On trouve dans L'Ève future une occurrence de ce terme écrit avec une majuscule et en italique (Villiers 1993, p. 149). Ces signes graphiques sont une manifestation du rôle décisif que le romancier accorde à cette composante.

8. Par exemple: "La voix — rieuse sur cette dernière parole — de l'être invisible " (Villiers 1993, p. 47) ; "“Une étincelle, et Hadaly apparaîtra!...”, dit la voix » (p. 48); "Une voix forte répondit au milieu de la chambre, bien qu'on ne vît personne" (p. 53) ; “"Qu'est-ce que tu veux, père?” disait la voix» (p. 55); "Est-ce que c'est le feu?... criait la voix tout effarée» (p. 110); “"J'attends, Monsieur Edison", répondit la voix, plus calme" (p. 111); " "Oui! j’y vais moi-même”, répondit la voix" (p. 111).

9. Le roman de Verne, dont traite notamment Tom Gunning (2001), focalise encore plus nettement l'attention sur la singularité vocale de l'être admiré et recréé par un dispositif audiovisuel. Pour le baron de Glotz, mélomane passionnément attaché à l'art d'une cantatrice, ce personnage féminin décédé n'était qu'une voix, 
«faute de laquelle il semblait qu'il n'aurait pu vivre» (Verne 1978, p. 135 et p. 137). Plutôt que d'être placée du côté des attributs physiques comme chez Villiers, la voix est foncièrement associée chez Verne à l'âme: la mort de l'artiste correspond exactement à la fin du chant, et le baron s'écrie lors de la seconde mort de la Stilla, au moment de la destruction de son simulacre phonographique: "Sa voix... sa voix!... répétait-il. Son âme... l'âme de la Stilla... Elle est brisée!» (p. 216).

10. Le couplage de différents médias est nécessairement provisoire selon cette définition, car Altman définit l'intermédialité comme une "crise de la médialité " qui se voit ensuite résorbée dans l'autonomisation médiatique.

11. J'adopte ici l'équivalent du terme "profilmique» des filmologues, proposé pour le domaine sonore par James Lastra (2000, p. 88). Ce terme a l'avantage, dans le contexte qui est le nôtre ici, de mettre l'accent sur la question de l'inscription (la "graphie») et de renvoyer explicitement à l'invention d'Edison.

12. Dans Le château des Carpathes, il est question d'appareils «si parfaits que la voix humaine n'y subissait aucune altération, ni dans son charme, ni dans sa pureté» (Verne 1978, p. 221).

13. Pour une critique argumentée de l'inaudibilité présupposée de la technologie sonore, voir Williams 1980.

14. Le romancier donne à ce propos la précision suivante: «Le procédé du Lampascope pouvait même, à l'aide d'une petite roue, modifier à tout instant l'expression des deux physionomies"(Villiers 1983, p. 95). Cet effet de transformation de la représentation visuelle est poussé dans L'Eve future jusqu'à la création d'un mouvement de type cinématographique. En outre, le lampascope est destiné dans L'Ėve future à la projection d'images photographiques et non dessinées, c'est-à-dire qu'il fait office de "stéréoscope". Notons que l'idée de coupler ce dispositif visuel avec le phonographe semble avoir circulé dès les premières présentations de ce dernier, comme en témoigne un article de 1878 qui se termine ainsi : "Il est déjà possible, à l'aide de procédés ingénieux, de projeter des photographies stéréoscopiques de gens sur un écran visible par tout un public. Ajoutez le phonographe parlant pour contrefaire leur voix, et il serait difficile de porter plus loin l'illusion d'une présence réelle» ("The Talking Phonograph", Engineering, 18 janvier 1878).

15. Notons que l'auteur de La machine animale ne dédaignait pas le modèle de "l'automate». Si l'on en croit Laurent Mannoni, le jeune Marey s'était déjà appliqué à construire un "magnifique polichinelle mécanique, dont les bras et les jambes remuent tout seuls"; plus tard, lorsque le physiologiste présente à l'Exposition internationale de la photographie (Paris, 20 avril 1892) les résultats de ses recherches sur la fixation des phases d'un mouvement, ses films sur celluloïd côtoient des "statues figurant le mouvement humain, étranges et abstraites épures de biomécanique (Mannoni, de Ferrière et Demenÿ 1997, p. 15-16 et p. 5). "L'épure" se situe par contre aux antipodes de la perfection du simulacre de l'androïde, ce qui révèle combien la démarche analytique du savant differe des intentions de cet homme de spectacle qu'est avant tout l'Edison de Villiers.

16. Affiche de C. Deaves reproduite dans Clair 1994 (p. 430-431). La machine n'y est pas identifiée, mais elle ressemble en tous points à l'Euphonia de Faber présentée au musée Barnum. Cette machine parlante est construite d'après le principe de l'imitation physiologique, tant en ce qui concerne son mécanisme (le clavier actionne la bouche, le larynx et la langue) que son apparence extérieure (il s'agit d'une poupée construite jusqu'au bassin, et affublée d'un vêtement). Voir au sujet de l'Euphonia les sources citées dans Lastra 2000 (p. 24-25 et p. 227).

17. À l'inverse de lord Ewald, qui ne perçoit aucunement la machinerie lorsqu'il converse avec l'Andréide, le narrateur-spectateur du texte de Schwob accède uniquement aux "coulisses" où les sons sont produits mécaniquement (il n’y a pas ici

L'Ève future et la série culturelle des "machines parlantes». Le statut singulier de la voix humaine au sein d'un dispositif audiovisuel 
d'imitation du faciès humain, la "gorge géante» se faisant l'expression démesurée d'une phobie du sexe féminin). L'émission vocale y est significativement précédée de "grincements de roues" et de "cris de fil en métal», bruits parasites qui exhibent l'origine mécanique de la voix. Le créateur de Schwob (1920, p. 151) affirme d'ailleurs s'être déshumanisé au contact de son invention: "[...] tant j'ai habité avec ma machine, je parle sans nuances comme elle; car la nuance appartient à l'âme, et je l'ai supprimée. » Notons également que la petite femme présentée par le démiurge comme étant «l'âme qui fait mouvoir le clavier» (p. 149) disparaît lorsque la machine explose, c'est-à-dire au moment où l'homme devient aphone. Ce finale témoigne d'une même association entre l'âme et la voix que celle suggérée chez Villiers, mais elle s'opère chez Schwob sur le mode de la dyspho(r/n)ie.

18. Bernadette Bensaude-Vincent et Christine Blondel (2002, p. 10) ont par exemple noté combien "les paradigmes dominants de la fin du XIX ${ }^{\mathrm{e}}$ " se prêtaient à "l'intégration de l'occulte dans la recherche scientifique». Les trois sciences dont ces auteurs relèvent l'importance dans ce contexte - psychologie, physiologie et physique - ont d'ailleurs toutes porté sur la voix (respectivement à travers l'étude de l'hystérie, du système phonatoire et de l'acoustique).

19. Si l'adhésion de Franz de Télek à la représentation audiovisuelle (pourtant dépourvue de mouvements) est si forte, c'est parce qu'elle est accompagnée d'une voix qu'il a reconnue et préalablement perçue dans le château sans avoir pu accéder à sa source. Jules Verne (1978, p. 188) a effectivement décrit auparavant comment Franz, prisonnier de la crypte du burg, entend les invitations répétées de la Stilla, en provenance de l'extérieur, à le suivre ("andiamo, mi cuore... andiamo...»), sans que la porte ne s'ouvre. Ce passage-clé met en scène un chant condamné à une répétition sans fin et détaché de tout ancrage spatial. Arrachée à sa deixis première (elle-même fictionnelle, puisqu'il s'agit d'une pièce d'opéra), la phrase n'est plus qu'une citation sans force illocutoire aucune: l'adresse ("mi cuore») est vide, l'injonction est vaine. Le tragique de cette porte qui refuse de s'ouvrir est celui de la perte d'un être cher que la reproduction phonographique se contente de recréer sous la forme d'une chimère.

20. "À cinq semaines, le bébé distingue la voix maternelle des autres voix, alors qu’il ne différencie pas encore le visage maternel des autres visages» (Anzieu 1976, p. 168).

\section{RÉFÉRENCES BIBLIOGRAPHIQUES}

Albera et Tortajada 2004: François Albera et Maria Tortajada, "L'épistémè "1900" ", dans André Gaudreault, Catherine Russell et Pierre Véronneau (dir.), Le cinématographe, nouvelle technologie du XXe siècle/The Cinema, A New Technology for the 20th Century, Lausanne, Payot Lausanne, 2004.

Altman 1980 : Rick Altman, «Introduction», Yale French Studies, n 60, 1980.

Altman 1989: Rick Altman, "Technologie et représentation: l'espace sonore», dans Jacques Aumont, André Gaudreault et Michel Marie (dir.), L'histoire du cinéma. Nouvelles approches, Paris, Publications de la Sorbonne, 1989.

Altman 2004: Rick Altman, Silent Film Sound, New York, Columbia University Press, 2004.

Anzieu 1976: Didier Anzieu, "L'enveloppe sonore du moi ", Nouvelle Revue de psychanalyse, $\mathrm{n}^{\circ} 13,1976$.

Arnoldy 2004: Édouard Arnoldy, Pour une histoire culturelle du cinéma. Au-devant de "scènes filmées", de "films chantants et parlants" et de comédies musicales, Liège, Céfal, 2004.

Bazin 1985: André Bazin, Qu’est-ce que le cinéma? [1946], Paris, Cerf, 1985. 
Bensaude-Vincent et Blondel 2002 : Bernadette Bensaude-Vincent et Christine Blondel, Des savants face à l'occulte, 1870-1940, Paris, Éditions de la Découverte, 2002.

Burch 1990 : Noël Burch, La lucarne de l'infini, Paris, Nathan, 1990.

Cavell 1999: Stanley Cavell, La projection du monde [1971], Paris, Belin, 1999.

Chapuis et Droz 1949: Alfred Chapuis et Edmond Droz, Les automates, Neuchâtel, Griffon, 1949.

Chateau 1986: Dominique Chateau, Le cinéma comme langage, Paris, Publications de la Sorbonne, 1986.

Chion 2004: Michel Chion, Un art sonore, le cinéma, Paris, Éditions des Cahiers du cinéma, 2004.

Clair 1994: Jean Clair, L'âme au corps. Arts et sciences 1793-1993, Paris/Milan, Réunion des Musées nationaux/Gallimard/Electa, 1994.

Comolli 1972: Jean-Louis Comolli, "Technique et idéologie 6 (II). Caméra, perspective, profondeur de champ", Cahiers du cinéma, n 241, 1972.

Coquio 1999: Catherine Coquio, "D'une espérance à l'essai, de son naufrage et d'une voix rescapée: Villiers de L'Isle-Adam, L'Ėve future», dans Pierre Brunel (dir.), L'homme artificiel, Paris, CNED-Didier, 1999.

Delacommune 1923: Charles Delacommune, «Le synchronisme de la parole, de la musique et des bruits au cinéma", dans Maurice Noverre, Septumia. Légende-mime (Mours antiques) [1922], Bordeaux/Paris, Bourlange et Mollat/Dion, 1923.

Deslandes 1966: Jacques Deslandes, Histoire comparée du cinéma, tome 1, Paris, Casterman, 1966.

Doyle 1981: Arthur Conan Doyle, Histoire du spiritisme [1927], Paris, Éditions du Rocher, 1981.

Gaudreault 1997: André Gaudreault, "Les vues cinématographiques selon Georges Méliès, ou : comment Mitry et Sadoul avaient peut-être raison d'avoir tort (même si c'est surtout Deslandes qu'il faut lire et relire)... ", dans Jacques Malthête et Michel Marie (dir.), Georges Méliès, liillusionniste fin de siècle?, Paris, Presses de la Sorbonne Nouvelle, 1997.

Grivel 1992: Charles Grivel, «La bouche cornue du phonographe» [1988], traduit en anglais dans D. Kahn et G. Whitehead (dir.), Wireless Imagination: Sound, Radio and the Avant-garde, Cambridge/London, MIT-Press, 1992.

Gunning 2001 : Tom Gunning, «Doing for the Eye What the Phonograph Does for the Ear", dans Richard Abel et Rick Altman (dir.), The Sounds of Early Cinema, Bloomington/Indianapolis, Indiana University Press, 2001.

Kircher 1966: Athanasius Kircher, Phonurgia Nova [1673], New York, Broude Brothers, 1966.

Ladoux 1989: René Ladoux, Le spiritisme, Paris, Cerf, 1989.

La Mettrie 1865: Julien Offroy de La Mettrie, L'homme machine [1748], Paris, Frédéric Henry, 1865.

Lastra 2000: James Lastra, Sound Technology and The American Cinema: Perception, Representation, Modernity, New York/Chichester, Columbia University Press, 2000.

Mannoni, de Ferrière et Demenÿ 1997 : Laurent Mannoni, Marc de Ferrière et Paul Demenÿ, Georges Demenÿ. Pionnier du cinéma, Douai, Pagine, 1997.

Metz 1993: Christian Metz, Le signifiant imaginaire [1977], Paris, Christian Bourgois, 1993.

Michelson 1984: Annette Michelson, "On the Eve of the Future: The Reasonable Facsimile and the Philosophical Toy", October, n ${ }^{\circ} 29,1984$.

L'Ève future et la série culturelle des "machines parlantes". Le statut singulier de la voix humaine au sein d'un dispositif audiovisuel 
Morin 1958 : Edgar Morin, Le cinéma ou l'homme imaginaire, Genève, Gonthier, 1958.

Noiray 1982 : Jacques Noiray, Le romancier et la machine. L'image de la machine dans le roman français (1850-1900), tome 2, Paris, J. Corti, 1982.

Pisano 2004: Giusy Pisano, Une archéologie du cinéma sonore, Paris, CNRS, 2004.

Pisano et Pozner 2005: Giusy Pisano et Valérie Pozner (dir.), Le muet a la parole, Paris, AFRHC, 2005.

Ponnau 2000 : Gwenhaël Ponnau, L'Ève future ou l'auvre en question, Paris, PUF, 2000 .

Schwob 1920: Marcel Schwob, "La machine à parler», dans Le roi au masque d'or [1892], Paris, G. Crès et Cie, 1920.

Vasse 1974: Denis Vasse, L'ombilic et la voix, Paris, Seuil, 1974.

Verne 1978 : Jules Verne, Le château des Carpathes [1892], Paris, Hachette, 1978.

Villiers 1983: Villiers de L'Isle-Adam, Contes cruels [1883], Paris, Gallimard, 1983.

Villiers 1993: Villiers de L'Isle-Adam, L'Ève future [1886], Paris, Gallimard, 1993.

Williams 1980 : Alan Williams, «Is Sound Recording Like a Language?», Yale French Studies, $n^{\circ} 60,1980$. 